\section{Conscious research in the} design process: a methodology for evaluating pre-existing conditions
| RESUMEN |

A principios de este siglo, Chile experimenta un cambio respecto a la protección de su patrimonio, la ciudadanía toma conciencia de concepto "patrimonio" y del deber como sociedad de protegerlo y el Estado requiere invertir adecuadamente los recursos dedicados a su protección. Desde la arquitectura surge la interrogante: jcómo abordar la intervención arquitectónica en una preexistencia de forma responsable, ética y pertinente? Intervenir debe ser una operación fundada, definida a través de un soporte histórico, técnico y arquitectónico, hacerlo consciente implica entender la pertinencia de la intervención. La elaboración de un diagnóstico y la definición de criterios de intervención asociados, permitirán el desarrollo de un proyecto consistente y eficiente, repercutiendo en la ciudad con una arquitectura coherente.

\section{| ABSTRACT |}

At the beginning of this century, Chile underwent a change regarding the protection of its heritage; citizens became aware of the concept of "heritage", of their duty as a society to protect this heritage, and the requirement of the State to properly invest resources intended for this protection. From an architectural point of view, the question arises: How do we address the architectural intervention in a pre-existent form in a responsible, ethical, and appropriate manner? Intervention should be an established operation, defined by historic, architectural and technical support; and doing it consciously implies an understanding of the relevance of the intervention. The development of a diagnosis and the definition of the corresponding criteria for intervention will allow the development of a consistent and efficient project impacting upon the city with coherent architecture.

\title{
Investigación consciente en el proceso proyectual: una metodología de evaluación de preexistencias
}

\section{INTRODUCCIÓN}

En atención al creciente interés y, muchas veces necesidad, de intervenir en nuestro patrimonio y la toma de conciencia de la importancia y responsabilidad que esto supone para los arquitectos, el propósito de este trabajo es presentar una metodología de evaluación de edificios que permita intervenir una preexistencia de forma responsable, ética y pertinente.

Dado que cada intervención patrimonial es única, esto es, edificios de distintas épocas, materialidades, sistemas constructivos, usos, estados de conservación, etc. la posibilidad de aplicar una metodología estándar se vuelve complejo. Lo que se pretende mostrar en este trabajo, es la flexibilidad que presenta dicho método para adaptarse a las distintas necesidades que presenta un edificio y sus demandas. Dicha adaptación no puede ser cualquiera y tal como se demostrará en el desarrollo, esta demanda una clara definición del encargo, entendido como nuestra definición de lo que se necesita evaluar, más allá de los requerimientos específicos de un mandante.

Esta pieza clave de la metodología es la que garantiza la definición de criterios de intervención que permitan el desarrollo de un proyecto consistente y eficiente, que prolongue la vida útil del edificio repercutiendo en la ciudad con una arquitectura coherente.

Por último, con este trabajo se pretende validar la interrelación entre enseñanza y su aplicación a nivel profesional, es solo gracias a este permanente intercambio de ideas, desde la academia a la práctica y viceversa, que se consigue el punto de equilibrio entre los aspectos teóricos necesarios de incorporar a toda investigación y su demostración en el ejercicio práctico de la profesión.

Arquitecto Universidad de Chile. Máster en Restauración Arquitectónica de la Universidad Politécnica de Madrid. Académico del Departamento de Arquitectura de la Facultad de Arquitectura y Urbanismo de la Universidad de Chile. Profesor del Postítulo de Conservación y Restauración Arquitectónica de la Universidad de Chile.

** Arquitecto Pontificia Universidad Católica de Chile. Máster en Restauración Arquitectónica de la Universidad Politécnica de Madrid. Arquitecto de la Secretaria Ejecutiva del Consejo de Monumentos Nacionales (CMN). Representante CMN en el Comité de Construcción Patrimonial del Instituto de la Construcción. Profesor del Postítulo de Conservación y Restauración Arquitectónica de la Universidad de Chile. 
1. Edificio calle Cochrane $N^{\circ} 25$ en su contexto (Fuente: autores).

\section{TRES VARIACIONES SOBRE UNA METODOLOGÍA DE EVALUACIÓN DE PREEXISTENCIAS}

Los casos que se exponen fueron realizados entre el año 2005 hasta la fecha y forman parte de una serie de trabajos realizados por nuestra oficina Paramento Limitada. Dentro de este período, coincidente al proceso académico que hemos desarrollado en el Postítulo de Conservación y Restauración de la Facultad de Arquitectura de la Universidad de Chile, se expusieron estos y otros casos de estudio para ser analizados y discutidos con el fin de reflexionar sobre el enfoque investigativo y el registro edificatorio realizado por nuestra oficina. Consecuentemente, se presentó sistemáticamente la aplicación de una metodología de evaluación en directa relación con requerimientos concretos y específicos por parte de diversos mandantes reales, presentando los propósitos de nuestros "encargos" en función de objetivos que esclarezcan la historia constructiva del edificio, su calidad constructiva y su estado de conservación.

La metodología o método aplicado tiene por objeto evaluar todos los componentes que forman parte de un edificio preexistente (estructura, fachadas, techumbre, componentes interiores, especialidades, etc.) determinando su estado de conservación o la patología constructiva $^{[1]}$ (Maldonado, 1999) que presenta, diagnosticando las causas de sus daños y estableciendo criterios de intervención.

A continuación, se exponen tres casos de estudio que explicarán el uso de una metodología, sus énfasis y resultados.
[1] Patología constructiva: Es la ciencia que estudia los daños constructivos de los edificios o de sus partes y su evolución en el tiempo (proceso patológico).

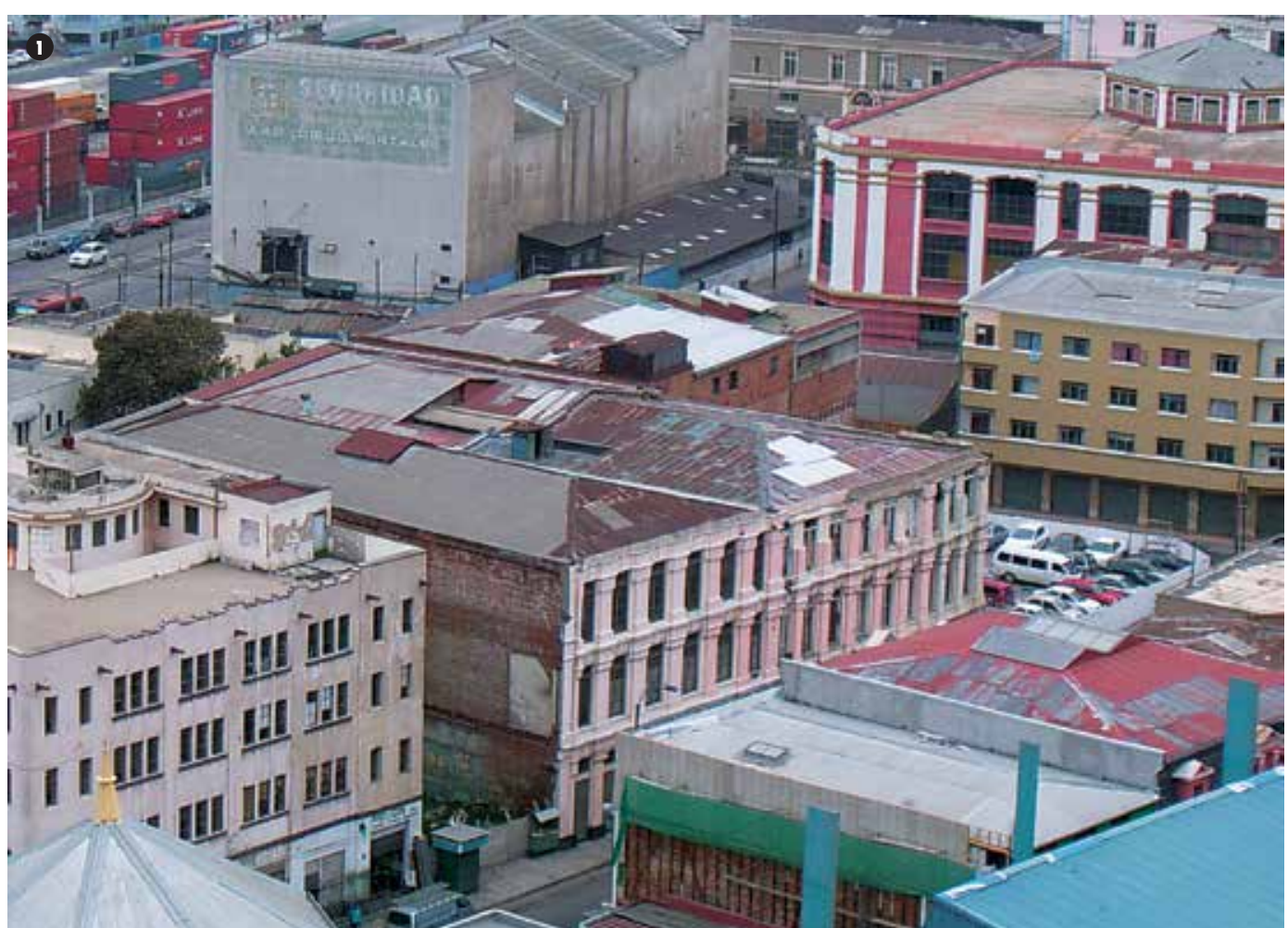

\section{CASO N 1}

EDIFICIO EN CALLE COCHRANE $N^{\circ} 25$, VALPARAÍSO (2005)

La Sociedad Inmobiliaria Cochrane Limitada, propietaria del inmueble, solicita una asesoría para las obras de rehabilitación del edificio con la finalidad de albergar un proyecto de arquitectura, con un uso programático de oficinas y comercio en un primer nivel y viviendas en los niveles superiores. Por lo que requieren de un reconocimiento del sistema estructural, sus componentes y respectivas funciones en el conjunto, definiendo los criterios de intervención en el edificio existente, basados en el estado actual de la estructura y sus posibilidades futuras. De tal forma, el objetivo del encargo es obtener información para el inicio del proyecto, consistente básicamente en un análisis histórico-constructivo y en un análisis estructural y tipológico.

Como antecedentes preliminares se contaba con planimetría elaborada por el mandante, memoria de cálculo de losa $7^{\text {er }}$ piso (1942) y proyecto de cálculo de bóveda del subterráneo (1966).

El edificio está emplazado en un terreno rectangular de aprox. $15 \mathrm{~m} \times 30 \mathrm{~m}$ entre medianeros a lo largo del edificio y con fachada a dos calles: Blanco y Cochrane. Tiene construidos tres pisos más un nivel de subterráneo, con un sistema estructural combinado de pórticos de hormigón armado y madera en su estructura interior. Los muros de fachada y medianeros son albañilería simple de ladrillo. Los entrepisos son de hormigón armado en el primer piso y envigados de madera en los dos siguientes. La estructura de cubierta es a base de cerchas de madera. Los tres niveles se conectan a través de escaleras interiores a excepción del subterráneo al que se accede desde el nivel de calle. Su morfología espacial es de planta libre asociada al uso de bodega y depósito.

El edificio cuyo origen se remonta hacia 1910 y 1912, fue realizado por encargo 
2. Fachada edificio, calle Blanco (Fuente: autores).

3. Interior, pórticos de madera $2^{\text {do }}$ piso (Fuente: autores).
4. Corte esquemático materialidades (Fuente: elaboración propia).
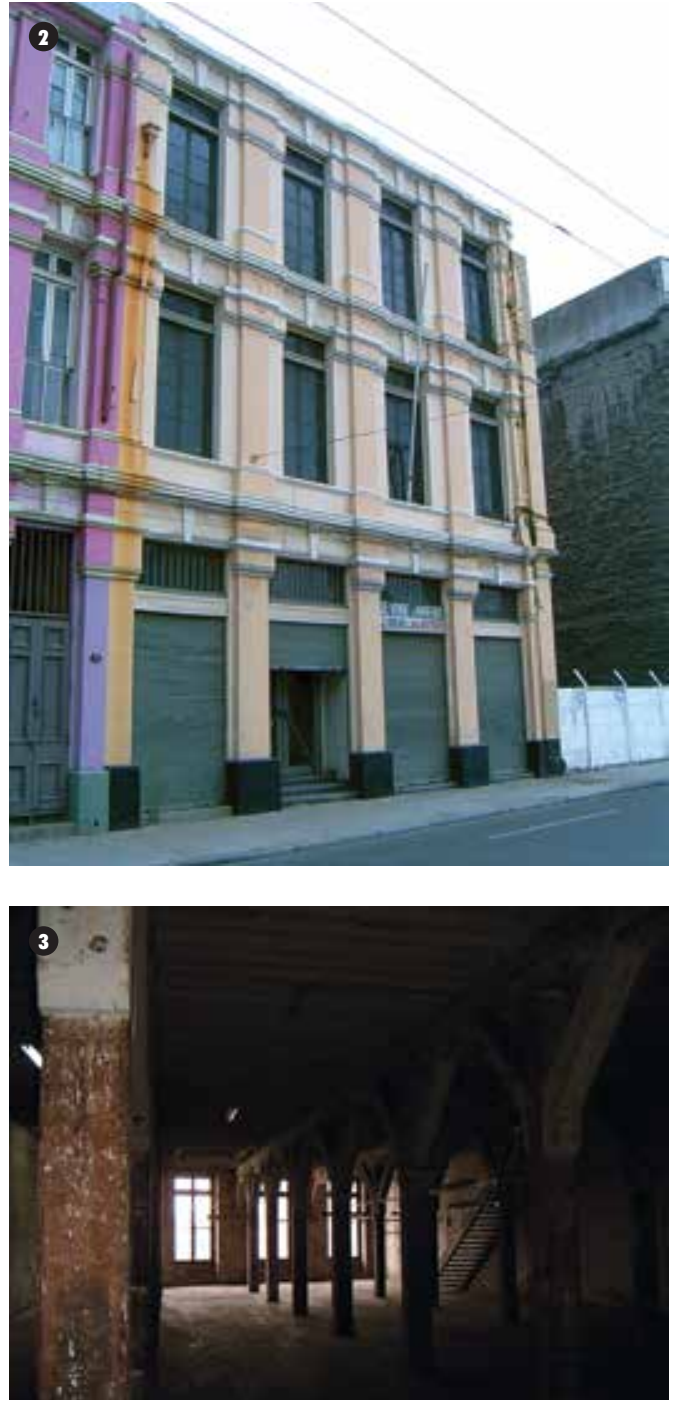

de un antiguo banco inglés y de acuerdo a los antecedentes históricos recopilados fue edificado en un sector de bodegas portuarias destruidas por el terremoto de 1906. En la década de 1940 fue adquirido por la Caja de Crédito Prendario donde se construyó la losa de hormigón armado del $1^{\text {er }}$ piso y una bóveda en el subterráneo. Hacia 1980, la propiedad fue comprada por un particular quien la arrendó como bodega.

Del análisis se desprenden dos grupos de conclusiones: Histórica-Constructiva y Estructural.

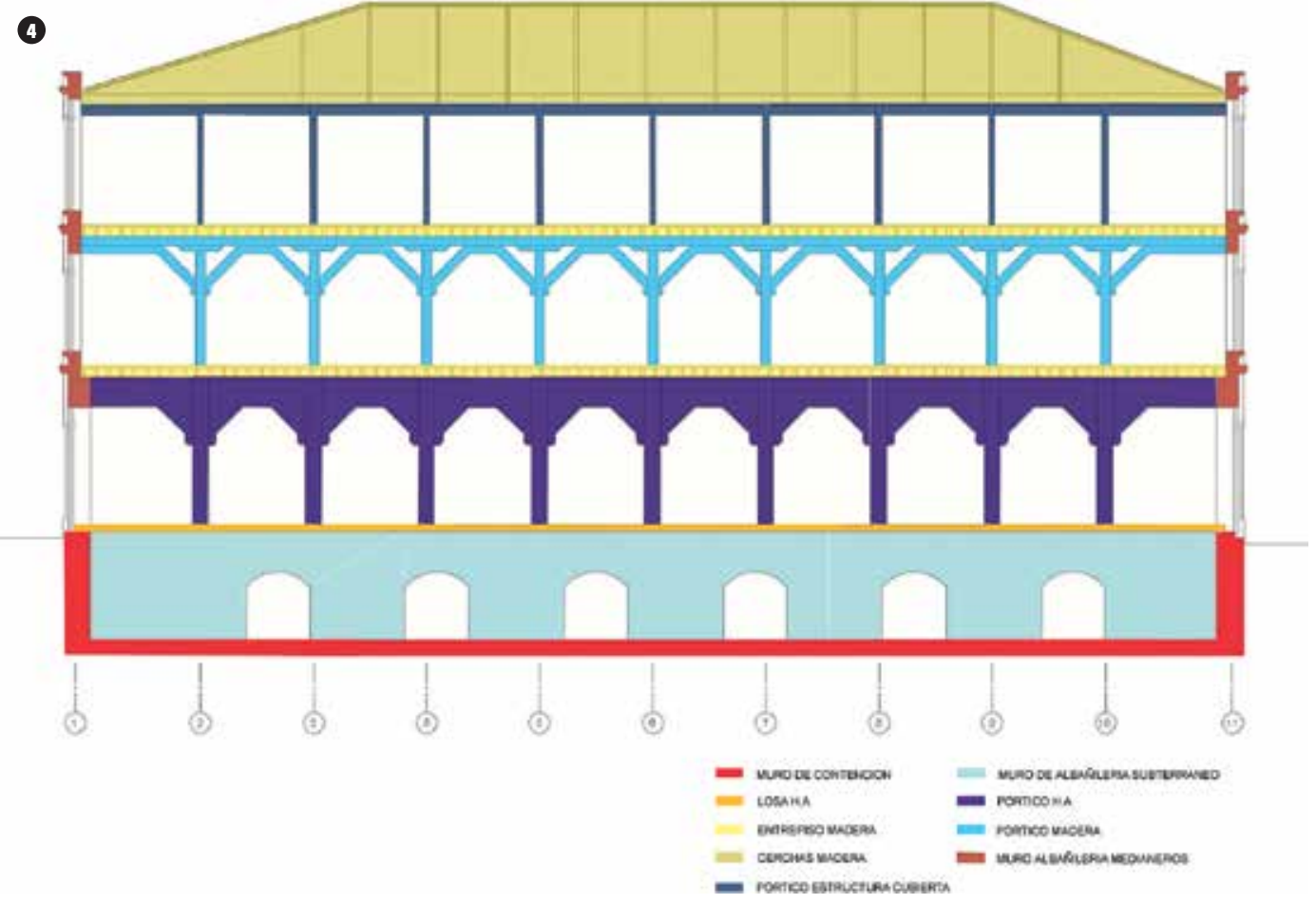

1. Histórico-Constructiva. Desde el siglo XIX, Valparaíso le ha ido ganando terreno al mar, creando calles que avanzan en esa dirección y generando grandes rellenos en la zona cercana al borde costero. Suponemos, en algún momento del siglo XIX, la aparición del muro de albañilería del eje central del subterráneo porque el nivel de altura que presentan los muros perimetrales del subterráneo $(3,60 \mathrm{~m})$ y la evidencia de ventanas en estos muros de fachada, hacen estimar la construcción del muro antes de 1906. La construcción del edificio fue entre los años 1910-1912, época de desarrollo histórico de las zonas portuarias y de las construcciones de apoyo al puerto que se hicieron después del terremoto de 1906. En este momento se habrían construido los pórticos de hormigón del subterráneo y $7^{\text {er }}$ piso, los pórticos de madera del $2^{\text {do }}$ piso, los pilares y viga de madera del $3^{\text {er }}$ piso y las estructuras de entrepisos y cubiertas de madera. Según documentación, la losa de hormigón armado se hizo el año 1942 en reemplazo del entrepiso existente entre el subterráneo y el $1^{\text {er }}$ piso y la bóveda de hormigón armado del subterráneo se construyó el año 1967 por la Caja de Crédito Prendario, propietaria del inmueble en ese momento. Dados los antecedentes obtenidos, se puede establecer que el edificio ha mantenido su uso a través del tiempo, cumpliendo funciones de bodega y almacenaje.

2. Estructural. El edificio se estructura en base a los dos muros medianeros longitudinales y los tres fuertes ejes interiores en el mismo sentido. No existen conexiones transversales, salvo los muros de fachadas. Existen entrepisos densos formando posibles diafragmas rígidos. Predomina la coincidencia de estructura en todos los niveles, la que va reduciendo sus secciones a medida que avanza en altura. Los pilares del eje central del $1^{\text {er }}, 2^{\text {do }}$ y $3^{\text {er }}$ piso, los que coinciden ordenadamente, descargan en forma aleatoria sobre el muro de albañilería del eje central del subterráneo, no correspondiendo ni con los tramos de muros ni con los arcos. 
Finalmente y debido al buen estado de conservación del inmueble, se recomendó que el proyecto de rehabilitación respete el valor histórico y representativo de una tipología propia de los puertos de nuestro país, manteniendo: la morfología del inmueble, la expresión arquitectónica de las fachadas y el rol urbano, como constituyente del barrio puerto (Muñoz et al, 2009). Asimismo, conserve la unidad estructural y su espacialidad original interior (planta libre) salvaguardando el muro central de albañilería de ladrillo del subterráneo y la estructura aporticada de hormigón y madera de los distintos niveles.

\section{CASO N 2}

\section{PLAN PILOTO DE MEJORAMIENTO DE VIVIENDAS EN EL CERRO SANTO DOMINGO, VALPARAÍSO (2007)}

\section{El Programa de Recuperación y Desarrollo} Urbano de Valparaíso (PRDUV), dependiente de la Subsecretaría de Desarrollo Regional del Ministerio del Interior, requirió de un plan de recuperación y rehabilitación de bienes muebles privados a través de un mejoramiento integral de 33 viviendas ${ }^{[2]}$ seleccionadas previamente. Para lo cual se planteó un encargo que tuvo por objeto dimensionar los daños y proponer a nivel de diseño completo -con las especificaciones técnicas correspondientes- los proyectos de mejoramiento para las viviendas contempladas en el plan, a fin de obtener su aprobación por la Dirección de Obras Municipales de Valparaíso.

A diferencia del caso anterior, la petición demandaba organizar levantamientos arquitectónico-constructivos para 33 viviendas agrupadas en 12 unidades o roles de avalúo ${ }^{[3]}$, desarrollar proyectos de arquitectura y especialidades para cada rol, informar periódicamente al mandante y consultar a los propietarios y comunicar a los residentes de las viviendas acerca de los cambios derivados de los proyectos de mejoramiento diseñados, que a petición del mandante, debían ser consensuados con ellos. En consecuencia, fue necesario contar con informes de diagnóstico y proyectos de mejoramiento para cada vivienda, informe final de gestión que dé cuenta de los resultados obtenidos y registro de proceso de desarrollo del trabajo.

Cabe señalar que las viviendas estudiadas son de máximo tres pisos de altura, con muros perimetrales e interiores formados por estructuras mixtas (madera, rellenos de adobillo y estucos de barro), revestidas exteriormente con planchas metálicas acanaladas y que, según el caso, presentan muros de contención de mampostería de piedra. Así pues, el Plan Piloto de Mejoramiento consistió en lo siguiente:

a. Levantamiento arquitectónico. Se realizaron levantamientos a once roles y la revisión planimétrica de un rol donde todas las construcciones tenían una data mayor de 70 años. Este levantamiento incluía las plantas de todos los niveles, cortes, elevaciones y el plano del terreno. Este último, presentó grandes dificultades de medición porque no se tenían claro los límites, sobre todo en casos en que los deslindes eran los taludes del cerro.

b. Informes de diagnóstico. Para cada vivienda, supuso la constatación del sistema estructural, cerramientos verticales (fachadas), estructuras de techumbre, componentes exteriores e instalaciones. Este análisis condujo a un diagnóstico y criterios de intervención. Los resultados de cada vivienda fueron resumidos en una ficha por rol que determinó los daños principales y los criterios de intervención en cuanto a los niveles de seguridad (lo que afecta a la estructura), funcionalidad (lo que afecta a las instalaciones) y aspecto de la edificación (lo que afecta a las terminaciones).

c. Proyectos. El proyecto de arquitectura se orientó principalmente a la ejecución de obras que resguardaran la seguridad de las edificaciones y que garantizaran su mantención en el tiempo. Coincidentemente estas suelen no estar al alcance de los propietarios, ya sea, constructiva y/o financieramente. De esta forma, se privilegiaron todas las faenas de refuerzos y consolidación estructural y luego actividades que evitaran su deterioro: instalaciones sanitarias (aguas lluvias, alcantarillado y agua potable) y eléctricas. Finalmente y si se veía afectada por las intervenciones a la estructura, se hicieron modificaciones interiores que mejoraran las condiciones de habitabilidad.

Los proyectos de cálculo se orientaron a conseguir una mejora sustancial en las condiciones de seguridad de la estructura. Para lo cual se trabajaron tres frentes de acción: contención de terreno, estructuras soportantes dañadas y deformaciones de piso. Para la contención de terreno se optó por reforzar por medio de mallas electrosoldadas y hormigón. Respecto a las estructuras dañadas, se estimó necesario reestructurar reemplazando piezas dañadas y rehaciendo las estructuras si era necesario. Y para las deformaciones, se planteó reforzar por medio de pilares y vigas metálicas buscando restituir la horizontalidad de las estructuras de piso. Respecto a la elaboración del presupuesto, se contaba con un financiamiento de $250 U{ }^{[4]}$ por vivienda -equivalentes a US\$12.080 aproximadamente- existiendo la posibilidad de ser flexibles con los montos entre roles, siempre y cuando no se excediera de la suma total establecida para el plan de mejoramiento, esto es 8.250 UF $=$ US\$398.640 (250 UF x 33 viviendas).

\footnotetext{
[2] Listado de 33 viviendas ubicados en el Cerro Santo Domingo seleccionados por el Programa de Recuperación y Desarrollo Urbano de Valparaíso.

3] Rol de avalúo: Número con el cual el Servicio de Impuestos Internos identifica una propiedad o bien raíz, el que es único en cada comuna.

4] Unidad de Fomento (UF): es una unidad financiera reajustable de acuerdo con la inflación (medida según el Índice de Precios al Consumidor o IPC) usada en la República de Chile.
} 
5. Fotografías de 12 roles analizados (Fuente: elaboración propia).

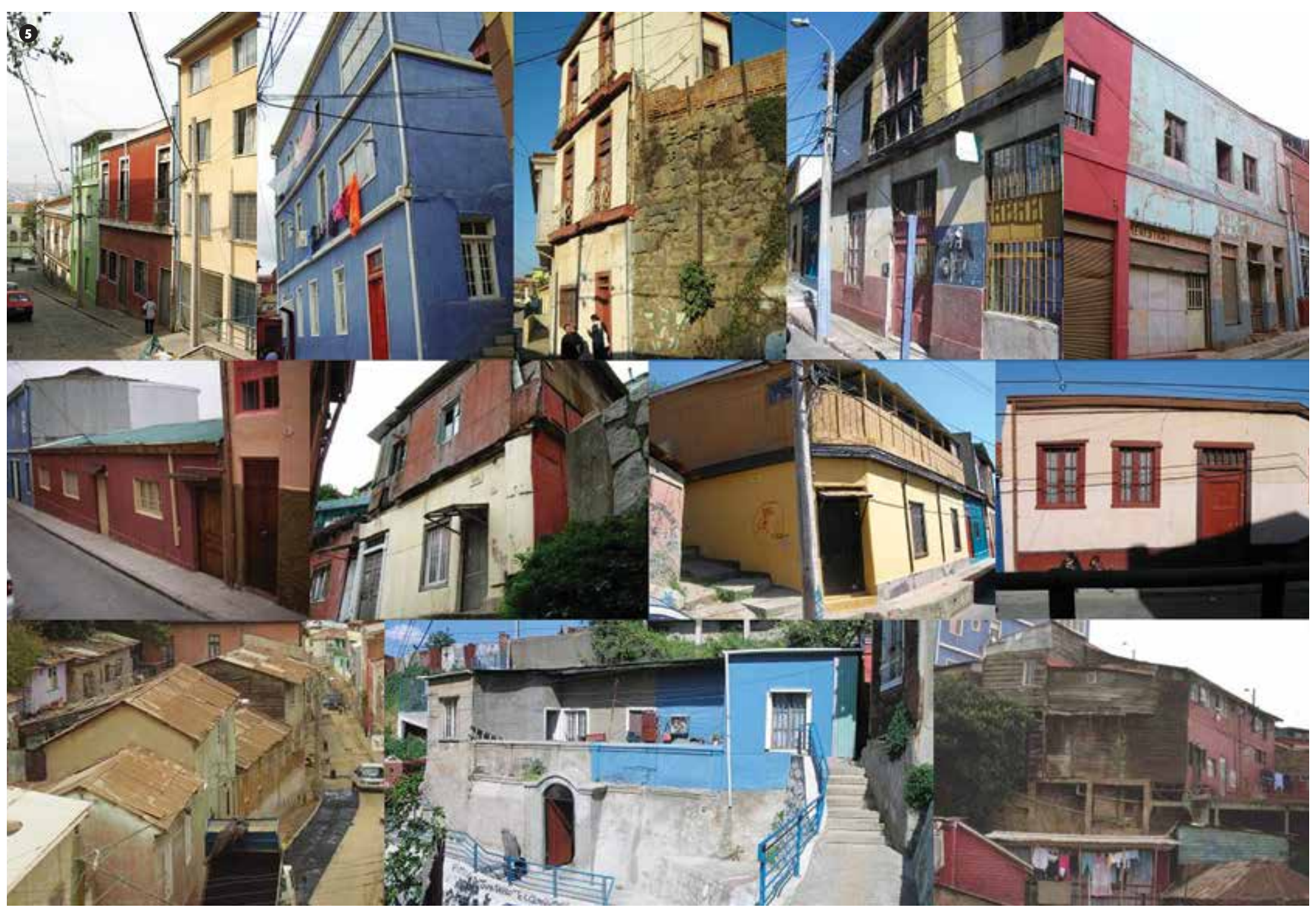

En relación a las actividades priorizadas en el proyecto de arquitectura, se cotizaron las partidas necesarias para las intervenciones de cada rol siendo fundamental la inclusión del control de organismos xilófagos,

desratización, readecuación de instalaciones eléctricas y mejoramiento de instalaciones sanitarias.

Por último, se definió el ingreso municipal en base a dos tipos de entrega al mandante los antecedentes básicos para obtener permiso municipal (proyecto de arquitectura, especificaciones técnicas y presupuestos) y los documentos complementarios al proyecto (informes de instalaciones sanitarias y eléctricas, proyecto de cálculo).

En consecuencia, se puede concluir que el proyecto denominado "plan de mejoramiento" planteaba, en su desarrollo inicial realizado por el mandante, efectuar remodelaciones en las viviendas; ampliaciones y modificaciones interiores considerables en intento de mejorar la funcionalidad, etc. Esta visión desvió la atención respecto a su objetivo inicial, ya que implicaba un desarrollo y financiamiento mayor al presupuesto asignado, con las respectivas responsabilidades que conllevaba. La metodología aplicada y la correcta definición del encargo determinaron que el parámetro del plan fuera de mejoramiento y no de reconstrucción ni remodelación. Esta aclaración permitió encauzar el desarrollo del proyecto de manera de ceñirse al presupuesto sin perder de vista los objetivos del plan, siendo estos básicamente, detener el deterioro de las edificaciones y mejorar las condiciones generales de las viviendas de manera de garantizar su permanencia en el tiempo. 
6. Corredor volumen principal (Fuente: autores).

Asimismo, la responsabilidad de los especialistas tenía directa relación con el alcance del proyecto y con el limitado presupuesto. Esto hace que, como no es posible plantear un proyecto nuevo, la participación de ingenieros estructurales y otros especialistas se limite a garantizar las condiciones de seguridad y el cumplimiento de la normativa específicamente de las intervenciones que se ejecutan, sin poder asegurarlo para el resto de la edificación.

\section{CASO N 3}

\section{CASONA DE LIRAY, COLINA (2011)}

La oficina de arquitectura + arquitectos necesita evaluar las condicionantes patrimoniales de la Casona de Liray, inmueble ubicado en la localidad de Liray perteneciente a la comuna de Colina y que se ubica dentro de la Zona de Conservación Histórica $(\mathrm{ZCH}){ }^{[5]}$ "Casas de Liray". Estos requerimientos se solicitaron debido a la futura construcción de un edificio de oficinas administrativas y un centro de información (data center) de una empresa de telecomunicaciones, dentro del predio donde se emplaza el inmueble.

En virtud de lo anterior, se formula un encargo que reúna los requerimientos del mandante y analice los valores asignados a la casona por la comunidad. Por esto, la metodología se orienta a realizar una descripción técnica constructiva del inmueble, un análisis histórico y social con énfasis en el reconocimiento de los valores recuperables y un catastro de elementos arquitectónicos rescatables con el objeto de ponerlos en valor.

Sobre la descripción técnica constructiva, este inmueble de un piso de altura está construido en bloques de adobe de entre 40 y $60 \mathrm{~cm}$ de espesor, sobre cimiento de ladrillo y estructura de madera para la techumbre. A pesar de no encontrar documentos escritos sobre su

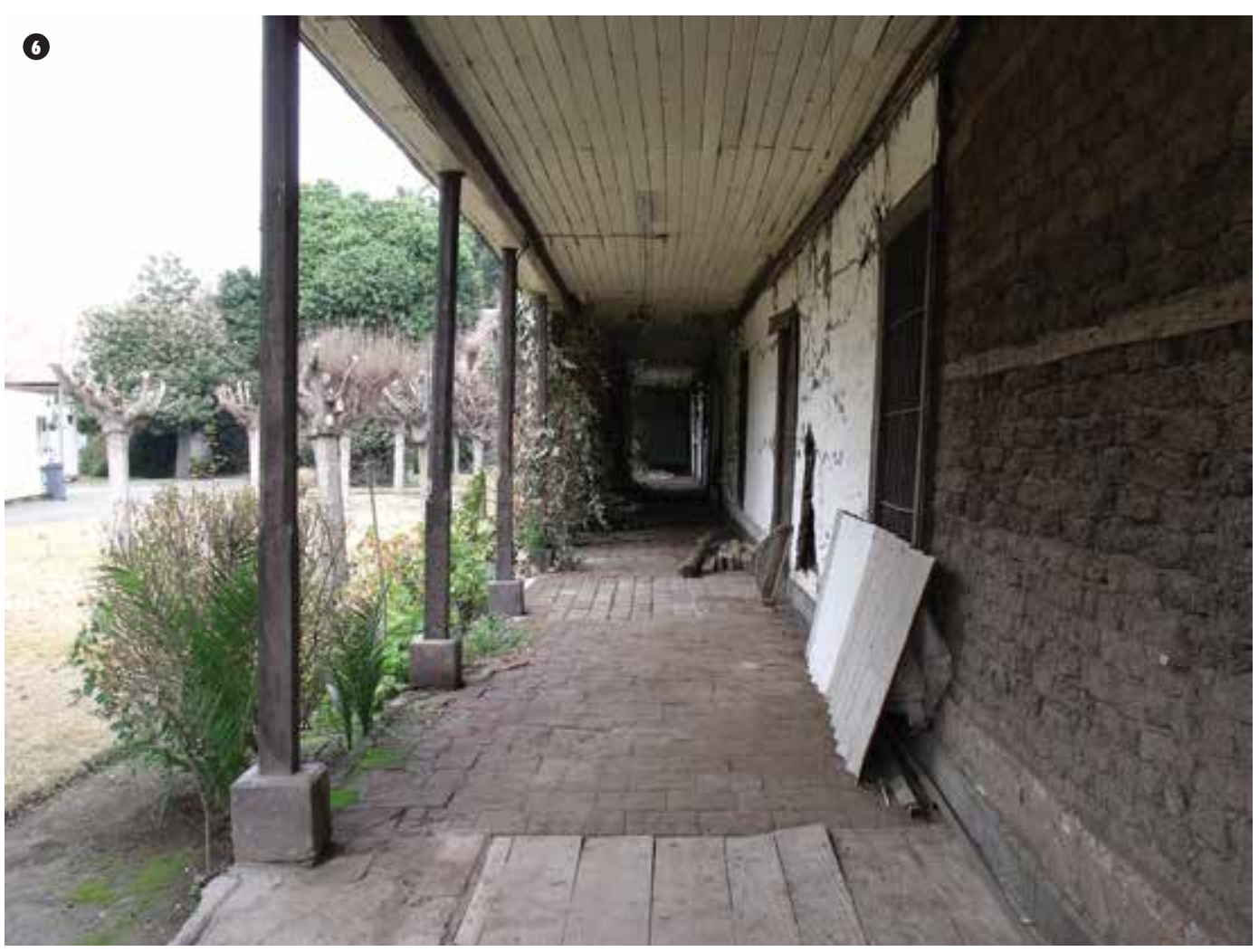

construcción, podemos afirmar que es una casona edificada en el siglo xIX con un uso principalmente productivo en las últimas décadas, esto de acuerdo a entrevistas al propietario y funcionarios municipales, además de constataciones en terreno tanto constructivas como territoriales del área de estudio.

Producto del terremoto del 27.02.2010[6], esta casona sufrió fuertes daños en su estructura por lo que la Municipalidad estableció su inhabitabilidad. En consecuencia y luego de realizar un diagnóstico general, se pudo observar varias zonas colapsadas evidenciándose lesiones en los sistemas de apertura de puertas, caída de techumbres y muros, desprendimientos de estucos y revoques, erosión y humedad generalizada, además de muchos desaplomes de muros y pilares. Asimismo, se detectó el uso de diferentes materialidades y diversas ampliaciones discordantes a la tipología y morfología de este tipo de arquitectura. Muchas de estas intervenciones son incompatibles al sistema constructivo del inmueble y determinan una menor calidad arquitectónica respecto a edificios de similar o mayor envergadura tanto constructiva como histórica.

Respecto al análisis histórico y social del inmueble, como primera actividad se investigaron numerosas publicaciones relacionadas a las casas patronales en Chile, identificando en este inmueble una localización

[5] Zona de Conservación Histórica (ZCH): es un área declarada por los Planes Reguladores Comunales de acuerdo a la Ley General de Urbanismo y Construcciones, Artículo 60 inciso $2^{\circ}$.

[6] El terremoto del 27 de febrero de 2010, de magnitud de 8,8 grados en la escala de Richter, afectó una extensa área del territorio nacional dañando en gran medida la arquitectura construida en tierra cruda, sistema constructivo propio del patrimonio protegido en Chile. 
7. Detalle puerta de hierro forjado (Fuente: autores).

8. Detalle constructivo muro (Fuente: autores)

9. Estado de deterioro del edificio (Fuente: autores) ortogonal a las vías de acceso, un cuerpo de una crujía y una ampliación posterior que agregó un volumen en forma de " $E$ " al cuerpo principal. Este emplazamiento genera dos patios rodeados por corredores exteriores y cubiertos: uno de acceso o de trabajo y un segundo espacio fraccionado que es el patio de servicio. Desde el punto de vista del valor social y acuerdo a información del último propietario, la casona ha sido un histórico lugar de trabajo y encuentro social para la comunidad local.

Por último, se solicitó un catastro de elementos recuperables con el fin de evaluar su reciclaje o puesta en valor conforme al futuro uso que tendrá el predio. Dentro de estos elementos podemos destacar los pavimentos existentes, las numerosas especies arbóreas del conjunto, puertas y ventanas y rejas metálicas de cierro.

En consecuencia, las conclusiones y recomendaciones para la puesta en valor fueron orientadas a recuperar el volumen central, considerando que es el cuerpo que representa mejor la configuración de la casona patronal; en atención al mal estado de conservación del volumen poniente y principalmente debido a la subdivisión predial que divide al edificio, se aconsejó desarmar toda esta extensión procurando preservar sus sobrecimientos y pavimentos con el fin mantener un registro de la evolución constructiva que ha tenido la casona; similar actividad se propuso para los volúmenes agregados que conforman la " $\mathrm{E}$ ", ya que son las áreas más colapsadas y claramente discordantes respecto a la morfología del edificio; y

finalmente en relación a las especies arbóreas, se recomendó categorizarlas de acuerdo a su antigüedad y valor paisajístico con el fin de ponerlas en valor e incorporarlas en el nuevo proyecto. Para todas las actividades anteriores, se aconsejó realizar un registro gráfico y escrito.

No obstante y ya que las condicionantes del proyecto consideraban la construcción de nuevos edificios, se sugirió como estrategia de recuperación de la casona la preservación del área donde se emplaza el edificio, es decir,
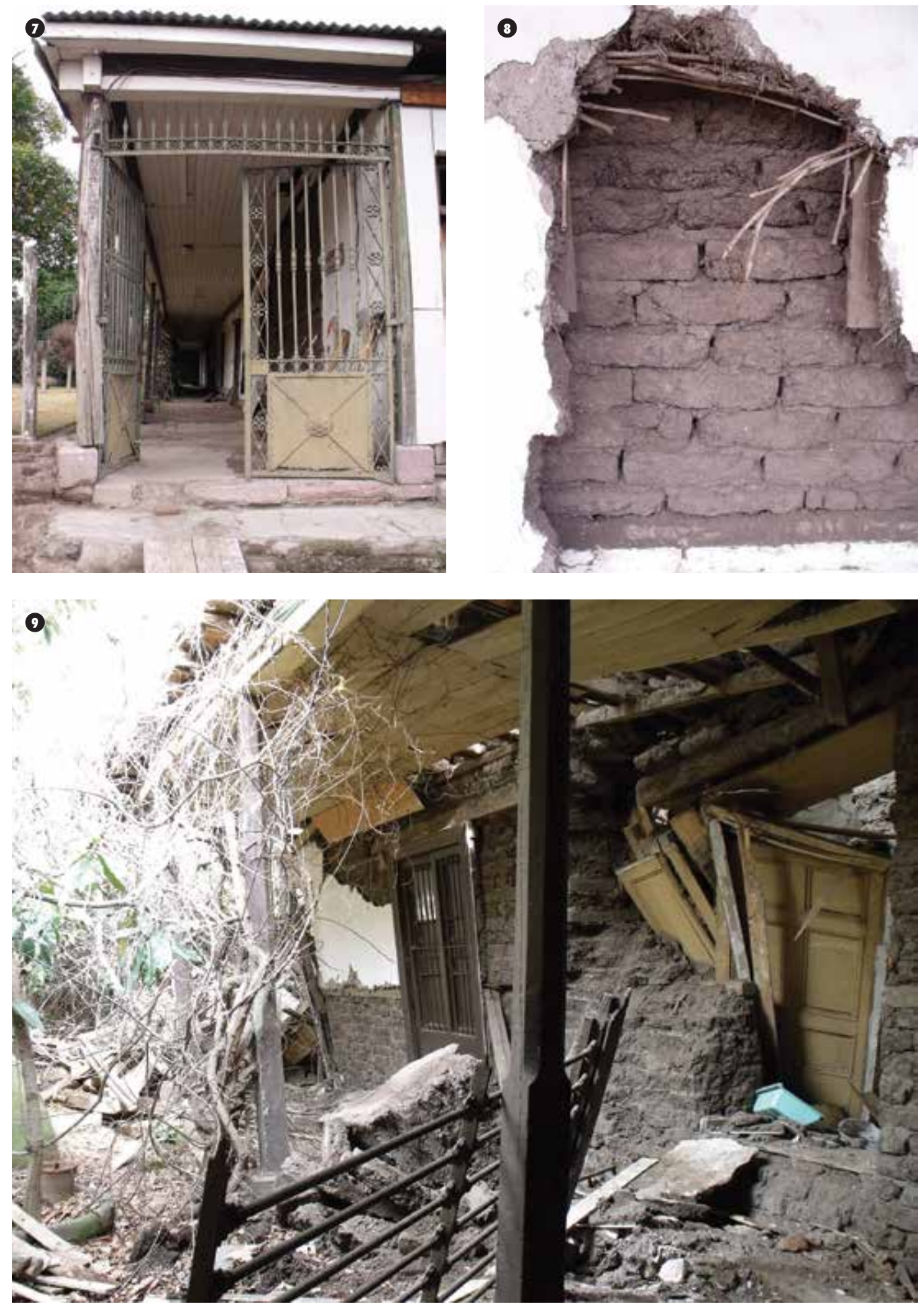
evitar construir sobre sus fundaciones a fin de mantener una huella que dé cuenta de su existencia. Esta huella o marca fundacional, puede ser jerarquizada manteniendo sus cimientos y pavimentos existentes y/o prospectando y poniendo en valor su subsuelo. Además, se sugiere recuperar la imagen del patio original de acceso, rearmando el portón de acceso y reconstruyendo el espacio con especies arbóreas que pongan en valor el edificio. En complemento, se planteó recuperar el concepto de patio jardín, es decir, preservar las especies valiosas y recomponer la función original de cada patio de acuerdo a su paisajismo original. Como existen rejas metálicas de valor, se estimó conveniente volver a reutilizarlas en algún acceso de la intervención o incorporarlas en la muestra museográfica que se hará en el nuevo edificio.

De la misma manera, para el proyecto de obra nueva se propone incorporar elementos morfológicos que componen la casona (corredor, patio principal y de servicios, etc.) y otros atributos espaciales de este tipo de arquitectura reinterpretando su imagen y función, utilizando un lenguaje contemporáneo y evitando falsos históricos.

\section{CONCLUSIONES}

La diversidad en los casos de estudio presentados, mandantes públicos o privados, edificio único o un conjunto de ellos, requerimientos muy amplios o muy específicos del cliente, etc., comprueban la flexibilidad de la metodología presentada y sus posibilidades de desarrollo.
La definición del encargo persigue la búsqueda del origen y el sentido de estos edificios preexistentes, en el intento de, a partir de sus propósitos originales o del de sus transformaciones históricas, volver a darle vida al edificio.

El uso de esta metodología siempre llevará a un diagnóstico como resultado, el que será pertinente a la arquitectura desde todos sus aspectos: histórico-social, técnico-constructivo, uso-función, etc., si es que ellos fueron, como debe ser, estudiados.

Si bien siempre habrá un diagnóstico, este no determina una única solución, el objetivo de la metodología es entregar herramientas para abrir las variables del problema, ampliando la visión de este. Tal como se presenta en la evolución de los casos de estudios, parece interesante la tendencia a entregar como resultado nuevas preguntas que orienten hacia la solución de las demandas de intervención.

Finalmente, serán los criterios de intervención definidos a partir de estas preguntas, los que precisen el cómo se debe intervenir una preexistencia o qué parámetros se deben respetar, en algunos casos estableciendo el tipo de intervención y en otros resguardando su integridad. Ellos permitirán la elaboración de un proyecto de intervención apropiado.

Por último, la permanente interacción entre la academia y el ejercicio profesional, nos ha entregado un espacio de relectura de nuestros procesos investigativos, instándonos a la revisión y análisis de los trabajos realizados, cerrando el ciclo con la necesaria evaluación que permite retroalimentar nuevos proyectos.

\section{BIBLIOGRAFÍA}

Indicadores Financieros.cl. Disponible [http://valoruf. $\mathrm{cl}$ que es la uf.html]

Maldonado, L. (1999). Toma de datos en patología constructiva. Tratado de Rehabilitación, Tomo

2: Metodología de la restauración y de la rehabilitación. Madrid. (España). Editorial Munilla-Lería, pp. 125-136.

Ministerio de Vivienda y Urbanismo, Chile. Circulares División de Desarrollo Urbano - DDU. Disponible [http://www.minvu.cl/ opensite_20070321153344.aspx].

Muñoz, G.; Sánchez, M.; Swinburn, V.; Swinburn, M. (2009). "Loft de la Aduana. Barrio Puerto". ARQ 73 Valparaíso, pp. 80-85.

Muñoz G.; Sánchez, M. (2005). Informe final. Asesoría obras de rehabilitación edificio Cochrane $N^{\circ} 25$. Santiago (Chile), autoedición.

Muñoz G.; Sánchez M. (2007). Informe final de gestión. Plan piloto de mejoramiento de viviendas, Cerro Santo Domingo, Valparaíso. Santiago (Chile), autoedición.

Muñoz G.; Sánchez M. (201 1). Informe arquitectónicosocial-histórico. Fundo Casas de Liray. Colina. Santiago (Chile), autoedición.

Servicio de Impuestos Internos, Chile. Diccionario básico tributario contable del Servicio de Impuestos Internos. Disponible [http://www.sii.cl/ diccionario_tributario/dicc_r.htm]. 